\title{
Virtual Reality Simulation in Peritoneal Dialysis Training: The Beginning of a New Era
}

\author{
Panagiota Zgoura $^{a}$ Daniel Hettich $^{b}$ Jonathan Natzel ${ }^{b}$ Fedai Özcan ${ }^{a}$ \\ Boris Kantzow ${ }^{b}$

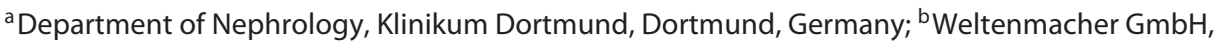 \\ VR Training-Application, Düsseldorf, Germany
}

\section{Keywords}

Peritoneal dialysis - Peritonitis rate $\cdot$ Virtual reality training program $\cdot$ Standardization $\cdot$ Education

\begin{abstract}
Background/Aim: Peritonitis rates in peritoneal dialysis (PD) vary considerably not only across countries but also between centers in the same country. Patient education has been shown to significantly reduce infection rates but up till now training lacks standardization with patients being trained using different methods and media (e.g., illustrations, videos). As a result, patients may be insufficiently experienced in performing $\mathrm{PD}$, which might be one of the causes for high peritonitis rates. To address these issues, we developed a PD training program based on virtual reality (VR). Methods: To become acquainted with the PD procedure, patients are equipped with a VR headset and controllers. They are presented with a virtual PD set, which simulates the feeling of sitting in front of a real PD set. The patient is enabled to run through the program as often as necessary to become familiarized with the whole PD procedure. The
\end{abstract}

\section{KARGER}

(c) 2018 S. Karger AG, Basel

E-Mail karger@karger.com

www.karger.com/bpu aim is to standardize, facilitate, and accelerate the individual learning process. To compare the effect of the applied training method to traditional training, a randomized controlled trial is underway. Conclusion: Previous studies on the effectiveness of learning showed that VR training applications are superior to traditional methods, such as text- or video-based training. However, no study has been undertaken in the context of dialysis. We believe that the implementation of VR training programs in clinical practice will be beneficial in improving the patient's proficiency, and thereby the quality and safety of PD.

(c) 2018 S. Karger AG, Basel

\section{Introduction}

Peritoneal dialysis (PD) is an effective and widely used renal replacement therapy. One of the most common complications linked to PD is peritonitis. Although peritonitis rates decreased during the last years and decades, there is continuous need for further reduction of infections [1]. Remarkably, there is a huge variation in the 
prevalence of peritonitis rates across different countries as well as across different centers in the same country [1]. In the last few decades, efforts for improvements primarily focused on refinement of PD materials. Recently, however, voices have been raised to shift the scientific focus toward standardization and optimization of training methods (cf. International Society of PD [ISPD] Guidelines) [1]. Currently, the training comprises a variation of methods, videos, and illustrations. This lack of standardization might be one of the reasons for the discrepancies in peritonitis rates across and within different countries.

This problem has been acknowledged by the ISPD which hence started to create a syllabus for teaching PD. Therefore, ISPD named 6 basic objectives for an improvement in PD training: Who should be the trainer? Who is the learner? What should be taught? Where should the training occur? What should be the duration of training? How should the patient be taught?

However, up till now many of these questions remain unanswered.

With focus on "How" and "What" should be taught?, we propose to add a new option to the traditional training methods which uses immersive and interactive media to achieve a more valid training quality. Therefore, we developed a training program based on virtual reality (VR) headsets and gamification elements: the patient finds himself in a virtual environment in which he is enabled to move and interact with certain stimuli in a similar way to the real world. The patient is accompanied by a virtual trainer who guides him through the process and assists him if he needs help. The program aims to familiarize the patient with the procedure of performing PD and intends to facilitate and accelerate the skill acquisition that is needed in order to execute the real PD process. The program is not only meant for patients but also for female and male nurses and trainees.

Our aim is to standardize each step of the whole PD procedure to improve the learning process and to control, store, and reproduce the results of each user. As a first step, we intend to implement this training concept in several training centers and hospitals. By this, we also plan to conduct a randomized controlled trial, in which we will further investigate the effectiveness of our VR application. Subsequently, we intend to develop VR applications for training at home.

VR gains more and more clinical importance in the medical area worldwide [2]. So far it has been used as a training simulation method mainly in endoscopy and surgery for students and trainees. There are some published trials that tested the validity of VR training simula-

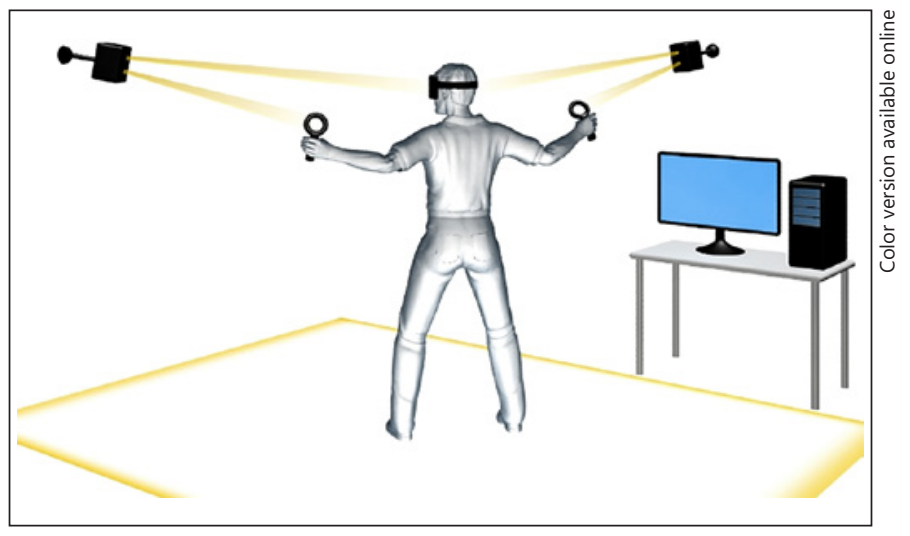

Fig. 1. Room-scale immersion and interaction with head-mounted display, controllers, and gaming engine (copyright Weltenmacher $\mathrm{GmbH}$ ).

tion. Thereby, VR has shown potential to improve existing trainings in endoscopy and laparoscopic training programs $[3,4]$. Additionally, studies showed that the learning effect is higher when we actively do things rather than only read, watch, or listen to them [5]. VR simulations combine all these different methods and thereby offer a better learning experience. Though studies have shown the potential of VR simulations, sample sizes, however, were limited in all of them [6-8]. Unfortunately, there is also no data existing about VR use in training programs concerning renal replacement therapy.

\section{Methods}

We developed a VR training program for patients as well as trainers and trainees, who must learn the whole procedure to conduct PD step by step. Our simulation offers a standardized learning protocol in which the different steps can be trained separately and put in the right order afterwards. Thereby, the training experience relies on VR technology which enables the user to feel like being in a different environment (immersion) and to interact with the items and stimuli around her/him like in the real world (interaction).

\section{Immersion}

To create the virtual environment, the user wears VR goggles (Head Mounted Display) which are tracked precisely in the room allowing him to visually and physically explore the surrounding virtual world (Fig. 1). For creating the virtual environment, it is required to collect user input from the Head Mounted Display and controllers in a continuous cycle to adjust the virtual world to real actions and to display it preferably without any delay [9]. It is also possible that a user can receive auditory and tactile feedback through headphones and controllers. For instance, sounds from different directions can be presented in the virtual space and simple tactile feedback can be send to the interaction devices (see below), while the user interacts with virtual objects. 


\section{Interaction}

The user uses controllers with his hands allowing him to grab and move virtual objects - to interact with the environment (Fig. 1). These interactions together with their associated computation are not pre-calculated, which allows real-time interaction, one of the main features of VR [10].

VR enables immersion and interaction in a way no other medium has been capable of, and it is thereby more engaging, impactful, and realistic than any other simulation technique. Thus, it is a new technology with huge potential for educational purposes.

In the PD training application, we are using 3 different modes to achieve the ISPD objectives (see above): PD.

1. Guided: User will be guided to all the different steps of

2. Free-run: User executes all the necessary steps and will get feedback on his performance after finishing the PD process.

Additionally, a distinction between different user groups with different needs, such as patient and trainer and also left- and righthanded users, is made.

The user can virtually train all the different steps that are required to perform the PD procedure safely and on his own. It is also possible to include automated pitfalls and barriers to put emphasis on the most common mistakes and train those steps even more intensely. Therefore, with our program, trainees and patients do not only experience the whole PD process but also get instructions and learn from the mistakes they make.

Furthermore, they can run through the program, completely or in parts, as often as it is necessary to internalize every single step (Fig. 2).

In addition, data about the individual learning progress can be collected throughout training sessions to improve the learning effectiveness or to document whether the training has been completed successfully. Users' progress, time, common mistakes, and other data can be stored in a digital file.

We are planning to test our VR training program against traditional training regarding the effectiveness of the tool and the learning progress of the user (e.g., number of mistakes made through full PD). In the long run, additional studies on the reduction of errors that users commit during the execution of the PD process are planned. Also, the relationship of the frequency of committed errors and occurrence of peritonitis should be tested.

The study design is still being worked out. It will be a randomized study including a large number of patients, trainees, and trainers. We expect to obtain the first results in 6 months.

\section{Discussion}

The improvement and standardization of education in PD has become a major issue during the last few years. Problems have been noted primarily in teaching patients before starting PD as a renal replacement therapy as well as in teaching novice trainees. Users are first trained with illustrations, video, and later they are instructed by nurses who teach patients and novice trainees face-to-face.

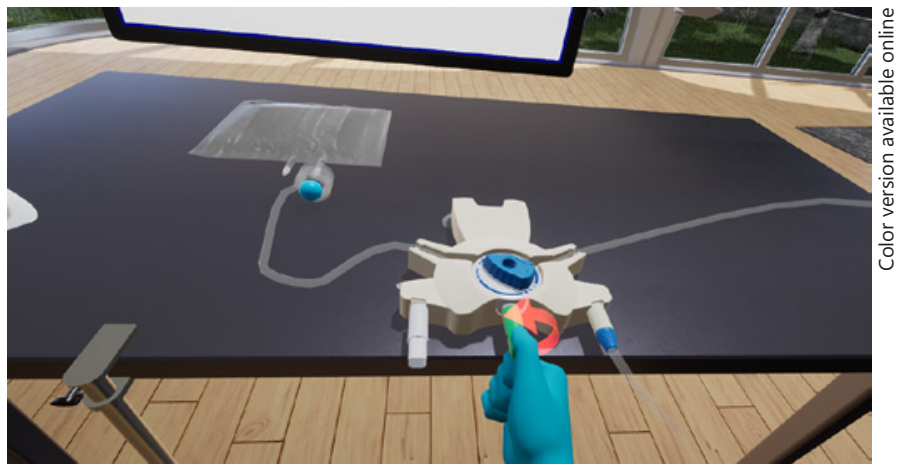

Fig. 2. User interaction with $P D$ gear within a virtual environment from the user's point of view.

So, training success depends on the abilities of the user and the trainer. The use of a VR simulation for educational purposes in $\mathrm{PD}$ guarantees a standardization of the whole process, and a faster and easier learning as well. Several studies showed that the use of VR in training programs improves skill acquisition amongst patients, trainers, and trainees. Maximum benefit has been seen amongst novice trainees [3]. Some reviews tried to determine the role of VR simulation within modern educational programs.

There is no existing data focused on VR use in nephrology so far. We can only comment on the findings referring to VR simulation training programs in endoscopy and laparoscopy for example. These results support the use of VR in training programs for endoscopy in several points. Unfortunately, the designs of the available studies are so different in terms of sample size, training time, participants, prior endoscopic experience, tasks included type of training, endpoints, and comparison between them in reviews is difficult. Despite all these factors, improvement in skill level has been demonstrated and the integration of VR simulation programs in endoscopy training curriculum has been advocated [11-14].

We are going to create a study design that will also give us an answer to the questions regarding the optimal exposure of VR simulation training. There are studies in which the exposure within formalized teaching settings was controlled. The total time trainees spent on VR simulator varied from 5 till $10 \mathrm{~h} \mathrm{[15-18].} \mathrm{One} \mathrm{study} \mathrm{showed}$ that there is a need of a 20-h exposure for a novice trainee to reach the level of an expert in colonoscopy [18]. In our case, we are going to have a look at how many hours of exposure users need to do the whole procedure of $\mathrm{PD}$ independently without help and mistakes. After that, we 
will test the exposure time novice trainees need to reach a well-defined expert level. We believe that VR training will reduce exposure time compared to the existing training methods along with costs for materials and training sessions.

Another limitation across the published studies was the variation in defining the "novice" and "experienced" trainees. There were no uniform criteria for the definition of advanced or top level expert.

Ferlitsch et al. [15] demonstrated that a training period of 3 weeks with $2 \mathrm{~h}$ per day improves the performance of beginners on the simulator. Virtual simulated endoscopy seems to be a tool that could provide a validated and much needed method for objective assessment of the user's skills too.

However, concerning all the mentioned points, there is a limited number of studies and participants. Regarding the effect of VR simulator training on clinical results, no relevant data has been published.

Yiannakopoulou et al. [19] evaluated VR training programs as beneficial based on Kirkpatrik's 4-level model. The levels are as follows:

Level 1: reactions measure how trainees react to the training program - face validity.

Level 2: learning assesses the extent to which trainees have made progress to performance - construct validity.

Level 3: behavior/transfer - measures the change in behavior due to the training program.

Level 4: results - assesses training in terms of clinical results, i.e., reduction in the number of complications [20].

Available data suggest that VR simulation training affects the trainees positively to perform better in terms of skill-based behavior and rule-based behavior. Skill-based behavior is that kind of behavior that takes place without conscious control. Rule-based behavior concerns task execution which is controlled by rules or procedures, for example, an operating protocol.

Thus, we need both types of behaviors for executing $\mathrm{PD}$, we are convinced that we can transfer these published findings about VR training simulators to our expected findings in regard to our case. All the critical points mentioned before should be inspected well and all doubts about them should be clarified. Furthermore, we want to use our training program in our clinical everyday life and provide clinical results in the next step.

\section{Conclusions}

VR simulation in training programs has been evaluated in endoscopy and laparoscopy teaching. Despite some limitations, these studies show evidenced benefit and advocate the use of VR simulation for educational purposes. VR simulation is capable of distinguishing between beginners and experts. It can detect progress and also the shift from beginners to experts' level. Because VR simulation training affects the users' performance in terms of skill-based and rule-based behaviors positively, we believe that it is an excellent tool for providing skills concerning the execution of PD. Right now, no clear model exists regarding the best way of VR training integration in the existing educational program. A combination of traditional teaching and VR simulator teaching will surely be a great benefit.

We are convinced that VR simulator training in PD alone is more effective and will reduce not only costs and time but also peritonitis rates in the long run. Above all, our training program offers the user standardization to a high level.

\section{References}

1 Figueiredo AE, Bernardini J, Bowes E, Hiramatsu M, Price V, Su C, Walker R, Brunier G: A syllabus for teaching peritoneal dialysis to patients and caregivers. Perit Dial Int 2016;36: 592-605.

2 VR Intelligence, Superdata: XR Industry Survey 2018, vol. 7, pp 1-20.

3 Cunningham M, Fernando B, Berlingieri P: The emerging role of screen based simulators in the training and assessment of colonoscopists. Frontline Gastroenterol 2010;1: 76-81.

4 Harpham-Lockyer L, Laskaratos FM, Berlingieri P, Epstein O: Role of virtual reality simu- lation in endoscopy training. World J Gastrointest Endosc 2015;7:1287-1294.

5 Dale E: Audiovisual Methods in Teaching. NY, Dryden Press, 1969.

6 Pellen M, Horgan L, Roger Barton J, Attwood S: Laparoscopic surgical skills assessment: can simulators replace experts? World J Surg 2009;33:440-447.

7 Moyano-Cuevas JL, Sanchez-Margallo FM, Sanchez-Peralta LF, Pagador JB, Enciso S, Sanchez-Gonzalez P, Gomez-Aguilera EJ, Uson-Gargallo J: Validation of SINERGIA as training tool: a randomized study to test the transfer of acquired basic psychomotor skills to LapMentor. Int J Comput Assist Radiol Surg 2011;6:839-846.

8 Sinitsky DM, Fernando B, Berlingieri P: Establishing a curriculum for the acquisition of laparoscopic psychomotor skills the virtual reality environment. Am J Surg 2012;204:367-376.e1.

9 Jerald J: The VR Book: Human-Centered Design for Virtual Reality, ed 1. New York, San Rafael, Association for Computing Machinery and Morgan \& Claypool Publishers (ACM Books Series) 2016, p 31.

10 Burdea GC, Coiffet P: Virtual Reality Technology, ed 2. Hoboken, Wiley-Inter-Science, 2003, p 2 . 
11 Clark JA, Volchok JA, Hazey JW, Sadighi PJ, Fanelli RD: Initial experience using an endoscopic simulator to train surgical residents in flexible endoscopy in a community medical center residency program. Curr Surg 2005;62: 59-63.

12 Bloom MB, Rawn CL, Salzberg AD, Krummel TM: Virtual reality applied to procedural testing: the next era. Ann Surg 2003;237:442448.

13 Ferlitsch A, Glauninger P, Gupper A, Schillinger M, Haefner M, Gangl A, Schoefl R: Evaluation of a virtual endoscopy simulator for training in gastrointestinal endoscopy. Endoscopy 2002;34:697-702.

14 Buzink SN, Koch AD, Heemskerk J, Botden SM, Goossens RH, de Ridder H, Schoon EJ,
Jakimowicz JJ: Acquiring basic endoscopy skills by training on the GI Mentor II. Surg Endosc 2007;21:1996-2003.

15 Ferlitsch A, Schoefl R, Puespoek A, Miehsler W, Schoeniger-Hekele M, Hofer H, Gangl A, Homoncik M: Effect of virtual endoscopy simulator training on performance of upper gastrointestinal endoscopy in patients: a randomized controlled trial. Endosc 2010;42: 1049-1056.

16 Shirai Y, Yoshida T, Shiraishi R, Okamoto T, Nakamura H, Harada T, Nishikawa J, Sakaida I: Prospective randomized study on the use of a computer-based endoscopic simulator for training in esophagogastroduodenoscopy. J Gastroenterol Hepatol 2008;23:10461050 .
17 Eversbusch A, Grantcharov TP: Learning curves and impact of psychomotor training on performance in simulated colonoscopy: a randomized trial using a virtual reality endoscopy trainer. Surg Endosc 2004;18:1514-1518.

18 Ahlberg G, Hultcrantz R, Jaramillo E, Lindblom A, Arvidsson D: Virtual reality colonoscopy simulation: a compulsory practice for the future colonoscopist? Endoscopy 2005;37: 1198-1204.

19 Yiannakopoulou E, Nikiteas N, Perrea D, Tsigris C: Virtual reality simulators and training in laparoscopic surgery. Int J Surg 2015; 13:60-64.

20 Hutchinson L: Evaluating and researching the effectiveness of educational interventions. BJM 1999;318:1267-1269. 